\title{
Informe del Secretario General de Naciones Unidas, Kofi Annan. "Un concepto más amplio de la libertad: desarrollo, seguridad y derechos humanos"
}

\begin{abstract}
A continuación se presenta un extracto del texto presentado por el Secretario General de Naciones Unidas a la Asamblea General, titulado Un concepto más amplio de la libertad: desarrollo, seguridad y derechos humanos, que se elaboró dentro del proceso de discusión de la transformación de Naciones Unidas. El texto completo de este documento está disponible en www. http://www.ohchr.org/spanish/bodies/hrcouncil/
\end{abstract}

\section{Libertad para vivir en dignidad}

127. En la Declaración del Milenio, los Estados Miembros afirmaron que no escatimarían esfuerzo alguno por promover la democracia y fortalecer el imperio de la ley y el respeto de todos los derechos humanos y las libertades fundamentales internacionalmente reconocidos. Reconocían así que, si bien la libertad para vivir sin miseria y la libertad para vivir sin temor son fundamentales, no resultan suficientes. Todos los seres humanos tienen derecho a ser tratados con dignidad y respeto.

128. La protección y la promoción de los valores universales del imperio del derecho, los derechos humanos y la democracia constituyen fines por sí mismas. También son imprescindibles para lograr un mundo de justicia, oportunidad y estabilidad. No habrá plan de seguridad ni campaña en pro del desarrollo que consiga sus objetivos si no reposa en el sólido fundamento del respeto por la dignidad humana.

129. En lo que se refiere a leyes promulgadas, ninguna generación ha heredado el tesoro que poseemos. Tenemos la fortuna de contar con lo que constituye una carta internacional de derechos humanos, que comprende normas admirables para proteger a los más débiles de nuestros semejantes, en particular las víctimas de los conflictos y las persecuciones. También contamos con un conjunto de normas internacionales sobre todos los temas, del comercio al derecho del mar, del terrorismo al medio ambiente y de las armas pequeñas a las armas de destrucción en masa. A través de una dura experiencia, la humanidad ha cobrado conciencia de la necesidad de incorporar en los acuerdos de paz disposiciones sobre los derechos humanos y el imperio del derecho y asegurarse de su cumplimiento. Una experiencia todavía más dura nos ha llevado a afrontar el hecho de que nunca debe permitirse que ningún principio jurídico -ni siquiera la soberanía- sirva de pretexto para el genocidio, los crímenes de lesa humanidad y el sufrimiento humano generalizado.

Este documento está disponible en: www.anuariocdh.uchile.cl 
130. Sin embargo, si no cumplimos lo acordado, nuestras declaraciones son palabras huecas. Si no pasamos a la acción, nuestras promesas son vanas. Los vecinos de las aldeas que se apiñan temerosos al oír el fragor de los bombardeos aéreos del gobierno o al ver aparecer a milicias asesinas no hallan consuelo en las palabras incumplidas de los Convenios de Ginebra, por no mencionar las solemnes promesas de "nunca más" que hizo la comunidad internacional cuando reflexionaba sobre los horrores de Rwanda hace un decenio. Los tratados que prohíben la tortura no sirven para consolar a los prisioneros que sufren abusos en manos de sus captores, especialmente si los mecanismos internacionales de derechos humanos permiten que los responsables se escondan detrás de sus amigos de las altas esferas. Una población hastiada de guerra a la que la firma de un acuerdo de paz infunde nuevas esperanzas pronto cae en la desesperación cuando, en lugar de percibir un progreso tangible hacia un gobierno respetuoso de la legalidad, ve cómo caudillos militares y cabecillas de bandas delictivas toman el poder y ocupan el lugar de las leyes. Las solemnes promesas de fortalecer la democracia en sus países que formularon todos los Estados en la Declaración del Milenio siguen siendo palabras vacías para quienes nunca han votado a sus dirigentes ni ven señal alguna de que estén cambiando las cosas.

131. Para impulsar una visión basada en un concepto más amplio de la libertad, las Naciones Unidas y sus Estados Miembros deben fortalecer el marco normativo que se ha promovido de modo tan admirable en los seis últimos decenios. Más importante aún es que adoptemos medidas concretas para reducir la tendencia a la aplicación selectiva de ese marco, el control arbitrario de su cumplimiento y su quebrantamiento con impunidad. Con esas medidas se daría nueva vida a los compromisos asumidos en la Declaración del Milenio.

132. Por consiguiente, creo que en 2005 deben adoptarse decisiones para contribuir a fortalecer el imperio de la ley en los planos internacional y nacional, potenciar la relevancia y la estructura de los mecanismos de derechos humanos de las Naciones Unidas y apoyar más directamente los esfuerzos por instituir y profundizar la democracia en las naciones de todo el mundo. También debemos avanzar hacia la meta de asumir la "responsabilidad de proteger" a las víctimas posibles o reales de atrocidades masivas y actuar en consecuencia. Ha llegado la hora de que los gobiernos deban rendir cuentas, ante sus ciudadanos y ante los demás gobiernos, del respeto a la dignidad de la persona, que con demasiada frecuencia se limitan a proclamar. Debemos pasar de la era de la formulación de leyes a la era de su cumplimiento. Nuestros principios declarados y nuestros intereses comunes no nos exigen menos.

\section{A. El imperio de la ley}

133. Estoy firmemente convencido de que toda nación que proclame el imperio de la ley en su territorio debe respetarlo más allá de sus fronteras y toda nación que insista en el imperio de la ley en el extranjero debe respetarlo en su propio territorio. En efecto, en la Declaración del Milenio 
se reafirmó el compromiso de todas las naciones con el imperio de la ley como marco primordial para promover la seguridad y la prosperidad de la humanidad. Sin embargo, en muchas partes hay gobiernos e individuos que no respetan la legalidad, muchas veces sin consecuencias para ellos, pero con consecuencias nefastas para los débiles y los vulnerables. En otros casos, los que ni siquiera fingen respetarla-como los grupos armados y los terroristas- pueden vulnerarla a causa de la debilidad de nuestras instituciones de mantenimiento de la paz y nuestros mecanismos de aplicación. El imperio de la ley como mero concepto no es suficiente. Deben promulgarse nuevas leyes, deben ponerse en práctica las que ya existen y las instituciones deben estar mejor equipadas para consolidarlo.

134. En ninguna esfera es la distancia entre la retórica y la realidad -entre las declaraciones y los hechos- tan marcada y tan perniciosa como en la del derecho internacional humanitario. Es intolerable que, cuando la comunidad internacional se encuentra frente a un caso de genocidio o de violación en masa de los derechos humanos, las Naciones Unidas se mantengan al margen y dejen que los acontecimientos sigan su curso hasta el final, con consecuencias desastrosas para muchos millares de personas inocentes. A lo largo de muchos años he llamado la atención de los Estados Miembros sobre esta cuestión. Con ocasión del décimo aniversario del genocidio de Rwanda, presenté un plan de acción de cinco puntos para prevenir el genocidio. En él se hace hincapié en la necesidad de actuar para prevenir los conflictos armados, adoptar medidas eficaces para proteger a los civiles, introducir disposiciones judiciales para acabar con la impunidad, establecer un mecanismo de alerta mediante el nombramiento de un Asesor Especial sobre la Prevención del Genocidio e intervenir de forma rápida y decisiva cuando ocurra o esté a punto de ocurrir un genocidio. Sin embargo, es necesario hacer mucho más por prevenir atrocidades y lograr que la comunidad internacional actúe con prontitud cuando se halle frente a vulneraciones masivas de los derechos humanos.

135. La Comisión Internacional sobre Intervención y Soberanía de los Estados y, más recientemente, el Grupo de alto nivel sobre las amenazas, los desafíos y el cambio, cuyos 16 miembros proceden de todas partes del mundo, aprobaron lo que denominaron "la norma que se está imponiendo en el sentido de que existe una responsabilidad internacional colectiva de proteger" (véase A/59/565, párr. 203). Aunque soy bien consciente de lo delicado de la cuestión, concuerdo totalmente con ese punto de vista. Debemos asumir la responsabilidad de proteger $y$, cuando sea necesario, debemos actuar en consecuencia. Esa responsabilidad recae, primordialmente, en cada Estado, cuya principal razón de ser y obligación es proteger a su población. Pero si las autoridades nacionales no están dispuestas a proteger a sus ciudadanos o no pueden hacerlo, se traslada a la comunidad internacional la responsabilidad de utilizar medios diplomáticos, humanitarios y de otro tipo para ayudar a proteger los derechos humanos y el bienestar de la población civil. Cuando esos métodos parecen ser insuficientes, el Consejo de Seguridad, puede si lo exigen las circunstancias, decidir adoptar medidas al amparo de la Carta 
de las Naciones Unidas, incluso, si es necesario, medidas coercitivas. En ese caso, como en otros, debe guiarse por los principios enunciados en la sección III supra.

136. El apoyo al imperio de la ley debe verse reforzado por la participación universal en los convenios multilaterales. En la actualidad, muchos Estados se mantienen fuera del marco constituido por los instrumentos multilaterales, lo que en algunos casos impide que entren en vigor convenios importantes. Cinco años atrás, ofrecí facilidades especiales a los Estados para que firmaran o ratificaran los tratados de los que soy depositario. La iniciativa dio muy buen resultado y desde entonces se han celebrado todos los años ceremonias de firma y ratificación de tratados. La ceremonia de este año estará centrada en 31 tratados multilaterales que nos servirán para responder mejor a los desafíos mundiales, especialmente en materia de derechos humanos, refugiados, terrorismo, delincuencia organizada y derecho del mar. Insto a los dirigentes a que ratifiquen y apliquen especialmente todos los tratados relativos a la protección de la población civil.

137. Para que todas nuestras iniciativas encaminadas a ayudar a las sociedades a superar un pasado violento obtengan los resultados deseados es fundamental contar con instituciones jurídicas y judiciales eficaces en el plano nacional. Sin embargo, las Naciones Unidas, las demás organizaciones internacionales y los gobiernos miembros siguen sin estar en las debidas condiciones para prestar apoyo a esas instituciones. Como indiqué en mi informe sobre el Estado de derecho y la justicia de transición en las sociedades que sufren o han sufrido conflictos (S/2004/616), carecemos de la necesaria capacidad de evaluación y planificación, tanto sobre el terreno como en la Sede. Por consiguiente, la asistencia que se presta es muchas veces improvisada, lenta e inadecuada para alcanzar el objetivo final. Para ayudar a las Naciones Unidas a desarrollar plenamente su potencial a ese respecto, tengo la intención de crear en la Oficina de Apoyo a la Consolidación de la Paz (véase la sección V infra) una dependencia de asistencia sobre el imperio de la ley, integrada en gran parte por personal existente del sistema de las Naciones Unidas, destinada a prestar asistencia a las iniciativas nacionales para restablecer la legalidad en sociedades que atraviesen o hayan atravesado un conflicto.

138. La justicia es un componente primordial del imperio de la ley. Se ha progresado extraordinariamente, como demuestran el establecimiento de la Corte Penal Internacional, la labor que cumplen los dos tribunales especiales para la ex Yugoslavia y Rwanda, la creación de un tribunal mixto en Sierra Leona, y según se espera, la próxima creación de otro en Camboya. Entre otras iniciativas importantes cabe mencionar las comisiones de expertos y de investigación, como las establecidas para Darfur, TimorLeste y Côte d'Ivoire. Sin embargo, los logros conseguidos en el derecho internacional humanitario siguen ensombrecidos por la impunidad, cuyas trágicas consecuencias en forma de atentados flagrantes y generalizados contra los derechos humanos continúan hasta el día de hoy. Para aumentar las posibilidades de indemnización para las víctimas 
de atrocidades y evitar nuevos horrores, exhorto a los Estados Miembros a que cooperen plenamente con la Corte Penal Internacional y otros tribunales internacionales o mixtos sobre crímenes de guerra, y que cuando se les solicite, les entreguen a las personas acusadas.

139. La Corte Internacional de Justicia ocupa un lugar central en el sistema internacional dedicado a arbitrar controversias entre Estados. En los últimos años, la Corte ha visto aumentar considerablemente su lista de causas y ha resuelto varias controversias, pero los recursos de que dispone siguen siendo escasos. Es necesario estudiar el modo de fortalecer la labor de la Corte. Insto a los Estados que todavía no lo hayan hecho a que consideren la posibilidad de reconocer la jurisdicción obligatoria de la Corte, como norma general de ser posible o, de lo contrario, al menos en situaciones concretas. También insto a todas las partes a que tengan presentes las facultades consultivas de la Corte y hagan un mayor uso de ellas. También deberían adoptarse medidas, con la cooperación de los Estados en litigio, para mejorar los métodos de trabajo de la Corte y reducir la duración de sus procedimientos.

\section{B. Derechos humanos}

140. Los derechos humanos son igualmente fundamentales para los pobres y para los ricos, y su protección es tan importante para la seguridad y prosperidad del mundo desarrollado como para la del mundo en desarrollo. Sería un error tratar los derechos humanos como si estuvieran contrapuestos a otros objetivos, como la seguridad y el desarrollo. Cederíamos terreno en la lucha contra los horrores de la pobreza extrema o el terrorismo si, en nuestros esfuerzos por acabar con esos males, negáramos los mismos derechos humanos de los que esos flagelos privan a la ciudadanía. Las estrategias basadas en la protección de los derechos humanos son fundamentales tanto para nuestra posición moral como para la eficacia práctica de nuestras acciones.

141. Desde su creación, las Naciones Unidas se han comprometido a luchar por un mundo de paz y justicia fundamentado en el respeto universal de los derechos humanos, misión reafirmada hace cinco años en la Declaración del Milenio. Sin embargo, el sistema de protección de los derechos humanos en el plano internacional se encuentra actualmente sometido a grandes presiones. Las Naciones Unidas necesitan cambiar para poder mantener su dedicación a largo plazo y a alto nivel a las cuestiones de derechos humanos en todos los aspectos de su labor.

142. Algunos cambios importantes ya están en marcha. Desde la Declaración del Milenio, los mecanismos de derechos humanos de las Naciones Unidas han ampliado su labor de protección, asistencia técnica y apoyo a las instituciones nacionales de derechos humanos, con el resultado de que las normas internacionales sobre derechos humanos se cumplen actualmente con mayor rigor en muchos países. El año pasado, puse en marcha la "Medida 2", un programa mundial destinado a preparar a los equipos interinstitucionales de las Naciones Unidas en los países para colaborar con los Estados Miembros, a solicitud de éstos, para 
revitalizar sus sistemas nacionales de promoción y protección de los derechos humanos. El programa necesita urgentemente más recursos y personal, incluida una mayor capacidad, en la Oficina del Alto Comisionado de las Naciones Unidas para los Derechos Humanos, para capacitar a los equipos que trabajan en los países.

143. La asistencia técnica y la consolidación de las instituciones a largo plazo no sirven de mucho cuando se infringe activamente el principio básico de la protección. Una mayor presencia de derechos humanos sobre el terreno en épocas de crisis permitiría que los organismos de las Naciones Unidas contaran con información puntual y, de ser necesario, serviría para llamar la atención con urgencia respecto de situaciones en las que fuera necesario intervenir.

144. La frecuencia cada vez mayor con que el Consejo de Seguridad invita a la Alta Comisionada a informarle sobre situaciones concretas demuestra que actualmente existe una mayor conciencia de la necesidad de tener presentes los derechos humanos en las resoluciones sobre la paz y la seguridad. La Alta Comisionada debe desempeñar un papel más activo en las deliberaciones del Consejo de Seguridad y en las de la Comisión de Consolidación de la Paz cuya creación se ha propuesto, prestando especial atención a la aplicación de las disposiciones pertinentes de las resoluciones del Consejo de Seguridad. Es más, los derechos humanos deben incorporarse en el proceso de examen y adopción de decisiones relativas a toda la labor de la Organización. El concepto de "incorporación" de los derechos humanos en esa labor ha sido objeto de mayor atención en los últimos años, pero todavía no se integrado suficientemente en decisiones importantes sobre cuestiones de políticas y asignación de recursos.

145. Todas estas observaciones apuntan a la necesidad de fortalecer la Oficina de la Alta Comisionada para los Derechos Humanos. Aunque la Alta Comisionada tiene ahora un papel más amplio que desempeñar en las respuestas a las crisis, el fomento de la capacidad nacional en materia de derechos humanos, el apoyo a los objetivos de desarrollo del Milenio y la prevención de conflictos, su Oficina sigue estando lamentablemente mal equipada para responder a la gran diversidad de problemas de derechos humanos que enfrenta la comunidad internacional. El compromiso de defender los derechos humanos proclamado por los Estados Miembros debe verse reflejado en la dotación de recursos que permitan fortalecer la capacidad de la Oficina para ejercer su mandato, que tiene importancia fundamental. He pedido a la Alta Comisionada que presente un plan de acción en los próximos 60 días.

146. La Alta Comisionada y su Oficina deben participar en toda la gama de actividades de las Naciones Unidas. Sin embargo, ese objetivo sólo podrá lograrse si nuestros mecanismos de derechos humanos cuentan con sólidos fundamentos intergubernamentales. Por consiguiente, en la sección $\mathrm{V}$ infra figura mi propuesta para transformar el órgano que debe ser el eje central del sistema de derechos humanos de las Naciones Unidas: la Comisión de Derechos Humanos. 
147. Por su parte, los órganos creados en virtud de tratados de derechos humanos también deben ser mucho más eficaces y reaccionar más rápidamente cuando se vulneran los derechos que tienen el mandato de defender. El sistema de los órganos creados en virtud de tratados sigue siendo poco conocido; su labor se ve dificultada por el hecho de que muchos Estados presentan sus informes fuera de plazo o sencillamente no los presentan, así como por la duplicación de los requisitos sobre la presentación de informes; y lo debilita todavía más la deficiente aplicación de sus recomendaciones. Deben elaborarse y aplicarse directrices armonizadas sobre la presentación de informes a todos los órganos creados en virtud de tratados a fin de que esos órganos puedan funcionar como un sistema unificado.

\section{Democracia}

148. En la Declaración Universal de Derechos Humanos ${ }^{19}$, aprobada por la Asamblea General en 1948, se enunciaban los principios fundamentales de la democracia. Desde el momento de su aprobación, la Declaración ha inspirado la redacción de constituciones en todas partes del mundo y ha contribuido en gran medida a que en el ámbito mundial finalmente se acepte la democracia como valor universal. El derecho de todas las personas, a elegir cómo serán gobernadas y quién debe gobernarlas debe ser un derecho innato y la realización universal de ese derecho debe ser uno de los principales objetivos de una Organización dedicada a promover un concepto más amplio de la libertad.

149. En la Declaración del Milenio, todos los Estados Miembros se comprometieron a aumentar su capacidad de aplicar los principios y las prácticas de la democracia. Ese mismo año, la Asamblea General aprobó una resolución sobre la promoción y la consolidación de la democracia ${ }^{20}$. Más de un centenar de países ya han firmado la Declaración de Varsovia sobre la Comunidad de Democracias (véase A/55/328, anexo I), y en 2002 esa Comunidad aprobó el Plan de Acción de Seúl (véase A/57/618, anexo I), en que se enunciaban los elementos fundamentales de la democracia representativa y se establecían medidas para promoverla. Las organizaciones regionales de muchas partes del mundo han hecho de la promoción de la democracia un componente básico de su labor, y el surgimiento de una fuerte comunidad de organizaciones de la sociedad civil de alcance mundial y regional que promueven la gobernanza democrática también es un hecho alentador. Todas esas iniciativas confirman el principio de que la democracia no pertenece a ningún país ni a ninguna región, sino que es un derecho universal.

150. Sin embargo, los compromisos deben ir acompañados de resultados concretos, y la protección de la democracia exige una labor de vigilancia. Las amenazas a la democracia de ningún modo han dejado de existir. Como ha podido verse una y otra vez, la transición a la democracia es delicada y difícil y puede sufrir graves retrocesos. Las Naciones Unidas prestan asistencia a los Estados Miembros proporcionando a las democracias incipientes ayuda y asesoramiento de carácter jurídico,
19 Resolución 217A (III) de la Asamblea General.

20 Resolución 55/96 de la Asamblea General. 
técnico y financiero. Por ejemplo, las Naciones Unidas han prestado apoyo concreto para la celebración de elecciones en un número cada vez mayor de países, con frecuencia en momentos decisivos de su historia: sólo en el último año sumaron más de una veintena, entre ellos el Afganistán, Palestina, el Iraq y Burundi. Del mismo modo, la labor de la Organización para mejorar la gobernanza en todo el mundo en desarrollo, promover el imperio de la ley y reconstruir las instituciones estatales en países devastados por la guerra es fundamental para asegurar que arraigue y perdure la democracia.

151. Aunque las Naciones Unidas hacen más que cualquier otra organización por promover y fortalecer las instituciones y las prácticas democráticas en todo el mundo, ese hecho es poco conocido. Los efectos de nuestra labor se ven disminuidos por el modo en que la dispersamos entre las distintas partes de nuestra burocracia. Ahora es preciso hacer encajar todas las piezas. Además, hay grandes lagunas en nuestra capacidad en varias esferas fundamentales. La Organización en su conjunto debe lograr una mejor coordinación y movilizar recursos con más eficacia. Las Naciones Unidas no deben limitarse a establecer normas, sino que deben ampliar la ayuda que prestan a sus miembros para seguir ampliando y afianzando las tendencias democráticas en todo el mundo. Con ese fin, apoyo la creación de un fondo para la democracia en las Naciones Unidas con objeto de prestar asistencia a los países que intenten establecer un régimen democrático o fortalecer el que tienen. Además, me propongo lograr que nuestras actividades en esa esfera estén más estrechamente coordinadas estableciendo una relación más explícita entre la labor de promoción de la gobernanza democrática que realiza el Programa de las Naciones Unidas para el Desarrollo y la que realiza la División de Asistencia Electoral del Departamento de Asuntos Políticos.

152. En las secciones II a IV me he referido a los desafíos, relacionados entre sí, que plantea la tarea de promover un concepto más amplio de la libertad en este nuevo siglo. También he indicado los que a mi juicio son los elementos fundamentales de nuestra respuesta colectiva, y concretamente muchas esferas en que creo que las Naciones Unidas deberían estar mejor equipadas para hacer la contribución que les corresponde. En la sección $\mathrm{V}$ me refiero en más detalle a las reformas concretas que juzgo necesarias para que nuestra Organización pueda desempeñar el papel que le incumbe en la formulación y aplicación de esa respuesta colectiva respecto de todas la cuestiones de interés mundial.

\section{Fortalecimiento de las Naciones Unidas}

153. En el presente informe sostengo que los principios y propósitos de las Naciones Unidas enunciados en la Carta siguen siendo tan válidos y pertinentes hoy como lo eran en 1945 y que el momento actual brinda una valiosa oportunidad de ponerlos en práctica. Sin embargo, aunque los propósitos deben ser firmes y los principios constantes, la práctica y la organización deben progresar al ritmo de los tiempos. Para ser un 
instrumento útil que ayude a los Estados Miembros y a los pueblos del mundo a responder a los desafíos descritos en las secciones II a IV, las Naciones Unidas deben adaptarse plenamente a las necesidades y circunstancias del siglo XXI. Deben estar abiertas no sólo a los Estados sino también a la sociedad civil, que desempeña, en los planos nacional e internacional, un papel cada vez más importante en los asuntos mundiales. Deben derivar su fortaleza de las asociaciones que establezcan y de su capacidad de hacer participar a sus asociados en coaliciones eficaces para impulsar el cambio en todas las esferas en que es necesario actuar para promover un concepto más amplio de la libertad.

154. Es evidente que nuestra Organización, como tal, fue concebida para otra época. También es evidente que no todas nuestras prácticas actuales están adaptadas a las necesidades de hoy. Es por ello que los Jefes de Estado y de Gobierno reconocieron, en la Declaración del Milenio, la necesidad de fortalecer las Naciones Unidas a fin de convertirlas en un instrumento más eficaz para el logro de sus objetivos prioritarios.

155. De hecho, desde que asumí el cargo de Secretario General en 1997, una de mis principales prioridades ha sido reformar las estructuras internas y la cultura de las Naciones Unidas para que la Organización sea más útil a sus Estados Miembros y a los pueblos del mundo. Es mucho lo que se ha conseguido ya. Hoy día, la Organización cuenta con estructuras racionalizadas, métodos de trabajo más eficaces y una mejor coordinación de sus distintos programas, y ha establecido relaciones de colaboración con la sociedad civil y el sector privado en muchas esferas. En las esferas económica y social, los objetivos de desarrollo del Milenio sirven actualmente de marco normativo común para todo el sistema de las Naciones Unidas y, en realidad, para toda la comunidad internacional que se ocupa del desarrollo. Actualmente, las misiones de mantenimiento de la paz están mucho mejor estructuradas que antes y tienen una visión más integrada de las múltiples labores que deben Ilevarse a cabo para prevenir la repetición de enfrentamientos y sentar las bases de una paz duradera. Además, hemos establecido alianzas estratégicas con una amplia gama de entidades no estatales que pueden hacer una importante contribución a la seguridad, la prosperidad y la libertad en el mundo.

156. Sin embargo, todavía es necesario hacer muchos más cambios. En la actualidad, la combinación de factores tales como las diferentes estructuras de gestión de los numerosos componentes del sistema, la superposición de mandatos y los mandatos que no reflejan las prioridades actuales sino las de otra época redunda en perjuicio de nuestra eficacia. Es fundamental conferir una autoridad real a los administradores para que puedan adaptar plenamente las actividades del sistema a los objetivos aprobados por los Estados Miembros, que espero que sean los expuestos en el presente informe. También debemos esforzarnos más por profesionalizar la Secretaría y hacer que tanto el personal como la administración rindan cuentas con mayor rigor sobre el desempeño de sus funciones. Debemos lograr asimismo una mayor coherencia, tanto 
entre la labor de los distintos representantes de las Naciones Unidas y las diferentes actividades que se llevan a cabo en cada país y en el sistema en su conjunto, especialmente en las esferas económica y social.

157. Sin embargo, para que sea eficaz, las reformas no pueden quedar confinadas a la rama ejecutiva. Es hora de infundir nueva vida también a los órganos intergubernamentales de las Naciones Unidas.

\section{A. Asamblea General}

158. Como se reafirmó en la Declaración del Milenio, la Asamblea General desempeña un papel central en su calidad de principal órgano deliberante, normativo y representativo de las Naciones Unidas. En particular, tiene autoridad para examinar y aprobar el presupuesto y elige a los miembros de los demás órganos deliberantes, incluido el Consejo de Seguridad. Por consiguiente, los Estados Miembros tienen motivos para estar preocupados por la pérdida de prestigio de la Asamblea y la menguante contribución de éste órgano a las actividades de la Organización. Esa tendencia debe invertirse, pero eso sólo ocurrirá si la Asamblea aumenta su eficacia.

159. En los últimos años, el número de resoluciones de la Asamblea General aprobadas por consenso ha ido aumentando constantemente. Ese aumento sería una buena señal si respondiera a una auténtica unidad de propósito entre los Estados Miembros para responder a los desafíos mundiales. Desgraciadamente, el consenso (interpretado muchas veces como una exigencia de unanimidad) se ha convertido en un fin en sí mismo. Se intenta conseguir primero dentro de cada grupo regional y luego en el plenario. Este sistema no ha resultado ser una forma eficaz de conciliar los intereses de los Estados Miembros. Más bien tiene el efecto de hacer que la Asamblea se limite a tratar generalidades, abandonando toda intención decidida de pasar a la acción. Los verdaderos debates que sí se celebran tienden a centrarse en el proceso más que en el fondo, y muchas de las Ilamadas decisiones reflejan tan sólo el mínimo común denominador de opiniones muy divergentes.

160. Los Estados Miembros concuerdan, como lo han hecho durante años, en que la Asamblea debe racionalizar sus procedimientos y estructuras para mejorar el proceso de deliberación y hacerlo más eficaz. Se han dado muchos pequeños pasos adelante. Un gran número de Estados Miembros ya han presentado nuevas propuestas para "revitalizar" la Asamblea. La Asamblea General debe adoptar ahora medidas decididas para racionalizar su labor y agilizar el proceso de deliberación, especialmente simplificando su programa, su estructura de comisiones y los procedimientos de los debates plenarios y la presentación de informes, y fortaleciendo el papel y la autoridad de su Presidente.

161. Actualmente, la Asamblea General tiene un amplio programa que comprende una variada gama de cuestiones que en muchos casos se superponen. La Asamblea debería dar una orientación más precisa a su programa sustantivo centrando su atención en las principales cuestiones de fondo del momento, como las migraciones internacionales y el 


\section{convenio general sobre el terrorismo que se debate desde hace tanto tiempo.}

162. También debería colaborar de forma mucho más activa con la sociedad civil, teniendo en cuenta que, tras un decenio de interacción en rápido aumento, actualmente la sociedad civil participa en la mayoría de las actividades de las Naciones Unidas. Es más, los objetivos de las Naciones Unidas sólo podrán alcanzarse si se cuenta con la plena participación de la sociedad civil y los gobiernos. El Grupo de personas eminentes encargado de examinar la relación entre las Naciones Unidas y la sociedad civil, que establecí en 2003, formuló muchas recomendaciones útiles para mejorar nuestra colaboración con la sociedad civil; he remitido su informe (véase A/58/817 y Corr.1) a la Asamblea General junto con mis opiniones. La Asamblea General debería adoptar medidas en relación con esas recomendaciones y establecer mecanismos que le permitan colaborar plenamente y en forma sistemática con la sociedad civil.

163. La Asamblea General también debe examinar su estructura de comisiones, la forma en que funcionan las comisiones y en que la Asamblea supervisa su labor y los resultados de esa labor. La Asamblea necesita un mecanismo para examinar las decisiones de sus comisiones a fin de no sobrecargar a la Organización con mandatos que carecen de fondos suficientes y perpetuar el problema actual de la microgestión del presupuesto y la asignación de puestos en la Secretaría. Si la Asamblea General no logra resolver esos problemas, no tendrá ni la claridad de objetivos ni la flexibilidad necesaria para prestar servicio a sus miembros con eficacia.

164. Debe quedar claro que nada de esto ocurrirá si los Estados Miembros no demuestran un interés auténtico en la Asamblea al más alto nivel y no insisten en que sus representantes participen en sus debates a fin de obtener resultados concretos y positivos. Si no lo hacen así, el funcionamiento de la Asamblea les seguirá pareciendo decepcionante, lo cual no deberá sorprenderlos.

\section{B. Los Consejos}

165. Los fundadores de las Naciones Unidas dotaron a la Organización de tres Consejos, cada uno de los cuales tenía importantes funciones en su esfera de competencia: el Consejo de Seguridad, el Consejo Económico y Social y el Consejo de Administración Fiduciaria. Con el tiempo, la distribución de funciones entre ellos ha resultado cada vez más desequilibrada: el Consejo de Seguridad ha ido afirmando progresivamente su autoridad y, especialmente desde el final de la guerra fría, ha disfrutado de una mayor unidad de propósito entre sus miembros permanentes, pero ha visto cuestionada esa autoridad por ser su composición anacrónica o insuficientemente representativa; el Consejo Económico y Social ha quedado muchas veces al margen de la gobernanza mundial en las esferas económica y social, y el Consejo de Administración Fiduciaria, habiendo cumplido satisfactoriamente sus 
funciones, se encuentra reducido en la actualidad a una existencia puramente formal.

166. Considero que debemos restablecer el equilibrio, de modo que haya tres Consejos que se ocupen, respectivamente, de: a) la paz y la seguridad internacionales, b) las cuestiones económicas y sociales, y c) los derechos humanos, cuya promoción, que ha sido uno de los objetivos de la Organización desde sus inicios, actualmente exige sin duda estructuras operacionales más eficaces. En conjunto, esos Consejos deberían encargarse de Ilevar adelante los planes que emanen de las cumbres y otras conferencias de los Estados Miembros, y deberían ser foros mundiales en los que puedan abordarse debidamente las cuestiones de la seguridad, el desarrollo y la justicia. Los dos primeros Consejos ya existen, desde luego, pero deben ser fortalecidos. El tercero exige una reestructuración de gran alcance y una potenciación del actual mecanismo de derechos humanos.

\section{Consejo de Seguridad}

167. En virtud de su adhesión a la Carta de las Naciones Unidas, todos los Estados Miembros reconocen que el Consejo de Seguridad tiene la responsabilidad primordial del mantenimiento de la paz y la seguridad internacionales y convienen en acatar sus decisiones. Por consiguiente, es de vital importancia, no sólo para la Organización sino para el mundo, que el Consejo esté en condiciones de desempeñar esa responsabilidad y que sus decisiones inspiren respeto a nivel mundial.

168. En la Declaración del Milenio, todos los Estados decidieron redoblar sus esfuerzos "por reformar ampliamente el Consejo de Seguridad en todos sus aspectos" (véase la resolución 55/2 de la Asamblea General, párr. 30). Esa decisión respondía al punto de vista, mantenido desde hacía tiempo por la mayoría, de que era necesario modificar la composición del Consejo para que fuera más ampliamente representativo de la comunidad internacional en su conjunto, así como de las realidades geopolíticas de nuestros días, y por consiguiente tuviera más legitimidad frente al mundo. Sus métodos de trabajo también deben ser más eficaces y transparentes. El Consejo no sólo debe ser más representativo, sino que también debe ser más capaz de adoptar medidas cuando sea necesario. La conciliación de esos dos imperativos es la prueba que debe superar toda propuesta de reforma.

169. Hace dos años, declaré que en mi opinión ninguna reforma de las Naciones Unidas estaría completa sin una reforma del Consejo de Seguridad. Sigo siendo de la misma opinión. El Consejo de Seguridad debe ser ampliamente representativo de las realidades del poder en el mundo actual. Por consiguiente, apoyo la postura expuesta en el informe del Grupo de alto nivel sobre las amenazas, los desafíos y el cambio (A) 59/565) en relación con las reformas del Consejo de Seguridad, a saber:

a) En cumplimiento del Artículo 23 de la Carta, deberían tener una mayor participación en el proceso de adopción de decisiones quienes más 
contribuyen a las Naciones Unidas desde el punto de vista financiero, militar o diplomático, especialmente en forma de cuotas para el presupuesto prorrateado de las Naciones Unidas, participación en operaciones de paz, aportaciones a actividades voluntarias de las Naciones Unidas en materia de seguridad y desarrollo y actividades diplomáticas en apoyo de los objetivos y mandatos de las Naciones Unidas. En el caso de los países desarrollados, el hecho de alcanzar la meta internacionalmente convenida de destinar el 0,7\% del PIB a la asistencia oficial para el desarrollo o de avanzar considerablemente hacia esa meta debe considerarse un criterio importante en cuanto a aportaciones se refiere;

b) Se debería incorporar en el proceso de adopción de decisiones a países que sean más representativos de la composición general de la Organización, especialmente del mundo en desarrollo;

c) Las reformas no deberían redundar en desmedro de la eficacia del Consejo de Seguridad;

d) Las reformas deberían hacer del Consejo un órgano más democrático y más responsable de su actuación.

170. Insto a los Estados Miembros a que estudien las dos posibilidades, modelos $A$ y $B$, que se proponen en ese informe (véase el recuadro 5), o cualquier otra propuesta viable en lo que respecta al número de miembros y el equilibrio que se hayan formulado sobre la base de uno u otro modelo. Los Estados Miembros deberían convenir en adoptar una decisión sobre esta importante cuestión antes de la celebración de la cumbre en septiembre de 2005. Desde luego, sería preferible que los Estados Miembros tomaran esa decisión vital por consenso, pero si no es posible, ello no debería servir de pretexto para postergarla.

Recuadro 5

\section{Reforma del Consejo de Seguridad: modelos A y B}

Según el modelo A habría seis nuevos puestos permanentes, no habría nuevos derechos de veto y habría tres nuevos puestos no permanentes de dos años de duración, divididos entre las grandes regiones según se indica a continuación:

\begin{tabular}{|c|c|c|c|c|c|}
\hline Región & $\begin{array}{l}\text { Número de } \\
\text { Estados }\end{array}$ & $\begin{array}{r}\text { Puestos } \\
\text { permanentes } \\
\text { (que se } \\
\text { mantienen }\end{array}$ & $\begin{array}{r}\text { Nuevos puestos } \\
\text { permanentes } \\
\text { (que se } \\
\text { proponen }\end{array}$ & $\begin{array}{r}\text { Puestos } \\
\text { un mandato } \\
\text { de dos años } \\
\text { (no renovable) } \\
\text { tue se proponen }\end{array}$ & Total \\
\hline África & 53 & 0 & 2 & 4 & 6 \\
\hline Asia y el Pacífico & 56 & 1 & 2 & 3 & 6 \\
\hline Europa & 47 & 3 & 1 & 2 & 6 \\
\hline América & 35 & 1 & 1 & 4 & 6 \\
\hline Total modelo A & 191 & 5 & 6 & 13 & 24 \\
\hline
\end{tabular}


Según el modelo B, no habría nuevos puestos permanentes pero se crearía una nueva categoría de ocho puestos con un mandato renovable de cuatro años y un nuevo puesto no permanente con un mandato de dos años (no renovable), divididos entre las grandes regiones según se indica a continuación:

\begin{tabular}{|c|c|c|c|c|c|}
\hline Región & $\begin{array}{r}\text { Número de } \\
\text { Estados }\end{array}$ & $\begin{array}{r}\text { Puestos } \\
\text { permanentes } \\
\text { (que se } \\
\text { mantienen }\end{array}$ & $\begin{array}{r}\text { Nuevos puestos } \\
\text { permanentes } \\
\text { (que se } \\
\text { proponen }\end{array}$ & $\begin{array}{r}\text { Puestos } \\
\text { un mandato } \\
\text { de dos años } \\
\text { (no renovable) } \\
\text { que se proponen }\end{array}$ & Total \\
\hline África & 53 & & 0 & 4 & 6 \\
\hline Asia y el Pacífico & 56 & & 1 & 3 & 6 \\
\hline Europa & 47 & & 3 & 1 & 6 \\
\hline América & 35 & & 1 & 3 & 6 \\
\hline Total modelo B & 191 & & 5 & 11 & 24 \\
\hline
\end{tabular}

\section{Consejo Económico y Social}

171. La Carta de las Naciones Unidas confiere al Consejo Económico y Social una serie de importantes funciones que comprenden la coordinación, el examen de polílticas y el diálogo sobre cuestiones normativas. La mayor parte de esas funciones parecen más fundamentales que nunca en esta época de globalización, en que las Naciones Unidas tienen un vasto programa de desarrollo emanado de las cumbres y conferencias celebradas en el decenio de 1990. Más que nunca, las Naciones Unidas deben ser capaces de formular y aplicar políticas en esa esfera de forma coherente. Por lo general se considera que las funciones del Consejo son extraordinariamente pertinentes para esas cuestiones, pero que todavía no se valoran como corresponde.

172. En 1945, los autores de la Carta no confirieron al Consejo Económico y Social la autoridad para tomar medidas coercitivas. Habiendo acordado en Bretton Woods el año anterior que se crearían poderosas instituciones financieras internacionales, y esperando que éstas se complementarían con una organización mundial del comercio, además de los distintos organismos especializados, tenían la evidente intención de que la adopción de decisiones económicas en el plano internacional estuviera descentralizada. Pero esto no hace más que realzar la importancia de las funciones que puede desempeñar el Consejo como coordinador, iniciador de conferencias, foro de diálogo sobre políticas y forjador de consensos. Es el único órgano de las Naciones Unidas al que la Carta asigna explícitamente el mandato de coordinar las actividades de los organismos especializados y mantener consultas con las organizaciones no gubernamentales. Además, tiene una red de comisiones orgánicas y regionales que funcionan bajo su égida y que se centran cada vez más en el cumplimiento de objetivos de desarrollo.

173. El Consejo Económico y Social ha hecho un buen uso de esas atribuciones en los últimos años, tendiendo puentes mediante la 
celebración anual de una reunión especial de alto nivel con las instituciones de las esferas comercial y financiera, por ejemplo, y tomando la iniciativa de establecer un Grupo de Tareas sobre la tecnología de la información y las comunicaciones, único en su género. También ha contribuido a vincular las cuestiones de seguridad y las de desarrollo estableciendo grupos especiales por países.

174. Esas iniciativas han contribuido a promover un mayor grado de coherencia y coordinación entre diferentes entidades, pero todavía subsisten visibles carencias que es necesario eliminar.

175. En primer lugar, existe una necesidad cada vez mayor de integrar y coordinar el programa de desarrollo de las Naciones Unidas emanado de las conferencias y cumbres mundiales y de examinar su aplicación. Para ese fin, el Consejo Económico y Social debería celebrar reuniones anuales de nivel ministerial sobre la marcha de la labor para alcanzar los objetivos de desarrollo acordados, en particular los objetivos de desarrollo del Milenio. Esas evaluaciones podrían basarse en exámenes recíprocos de los informes preparados por los Estados Miembros, con el apoyo de los organismos de las Naciones Unidas y las comisiones regionales.

176. En segundo lugar, existe la necesidad de examinar las tendencias de la cooperación internacional para el desarrollo, promover una mayor coherencia de las actividades de desarrollo de las diferentes entidades y fortalecer los vínculos entre la labor normativa y la labor operacional del sistema de las Naciones Unidas. Para eliminar esa carencia, el Consejo Económico y Social debería servir de foro de alto nivel sobre la cooperación para el desarrollo. Ese foro podría celebrarse cada dos años adaptando la serie de sesiones de alto nivel del Consejo.

177. En tercer lugar, existe la necesidad de abordar los desafíos, las amenazas y las crisis en las esferas económica y social en el momento en que ocurren. Para ello, el Consejo debería celebrar reuniones oportunamente, cuando resultara necesario, para evaluar las amenazas al desarrollo, como las hambrunas, las epidemias y los grandes desastres naturales, y promover respuestas coordinadas para afrontarlas.

178. En cuarto lugar, existe la necesidad de vigilar y abordar sistemáticamente las dimensiones económicas y sociales de los conflictos. El Consejo Económico y Social ha intentado atender esa necesidad estableciendo grupos especiales de asesoramiento por países. Sin embargo, habida cuenta de la magnitud y la dificultad de la labor de recuperación, reconstrucción y reconciliación a largo plazo, las medidas tomadas caso por caso no resultan suficientes. El Consejo Económico y Social debería institucionalizar su labor de gestión de las situaciones posteriores a los conflictos colaborando con la Comisión de Consolidación de la Paz que se ha propuesto. También debería reforzar sus vínculos con el Consejo de Seguridad para promover medidas de prevención de carácter estructural.

179. Por último, aunque las funciones normativas y de formulación de estrategias del Consejo Económico y Social son claramente distintas de 
${ }^{21}$ Resolución 2200A (XXI) de la Asamblea General. las funciones directivas y de formulación de políticas que desempeñan los órganos rectores de las diferentes instituciones internacionales, tengo la esperanza de que, a medida que el Consejo empiece a consolidar su liderazgo en la promoción de un programa mundial de desarrollo, pueda proporcionar orientación a los distintos órganos intergubernamentales que intervienen en esa esfera en todo el sistema de las Naciones Unidas.

180. El cumplimiento de todas esas recomendaciones exigiría que el Consejo Económico y Social funcionara con una estructura nueva y más flexible, sin limitarse necesariamente al actual calendario anual de "series de sesiones" y "períodos de sesiones sustantivos". Además, el Consejo necesita un mecanismo intergubernamental eficaz, eficiente y representativo para fomentar la participación de sus contrapartes en las instituciones que se ocupan de las finanzas y el comercio. Ese objetivo podría lograrse ampliando su Mesa o estableciendo un comité ejecutivo con una composición regionalmente equilibrada.

\section{Propuesta de crear un Consejo de Derechos Humanos}

181. La Comisión de Derechos Humanos ha proporcionado a la comunidad internacional un marco universal de derechos humanos que comprende la Declaración Universal de Derechos Humanos, los dos Pactos Internacionales ${ }^{21}$ y otros tratados fundamentales en la materia. En su período de sesiones anual, la Comisión señala a la atención del público cuestiones y debates en relación con los derechos humanos, sirve de foro para la elaboración de las políticas de derechos humanos de las Naciones Unidas y establece un sistema único de procedimientos especiales independientes y de expertos para observar y analizar el respeto de los derechos humanos por temas y por países. La estrecha relación de la Comisión con centenares de organizaciones de la sociedad civil brinda una oportunidad de colaboración con la sociedad civil que no existe en otras instancias.

182. Sin embargo, la disminución de la credibilidad y el profesionalismo de la Comisión de Derechos Humanos ha menoscabado progresivamente la capacidad de la Comisión para desempeñar sus funciones. En particular, ha habido Estados que se han hecho miembros de la Comisión no para afianzar los derechos humanos sino para protegerse contra las críticas o para criticar a otros. Esas tendencias han tenido como resultado la acumulación de un déficit de credibilidad que empaña la reputación de todo el sistema de las Naciones Unidas.

183. Para que las Naciones Unidas cumplan las expectativas de los hombres y las mujeres de todo el mundo $y$, de hecho, para que la Organización asigne a la causa de los derechos humanos la misma importancia que a las causas de la seguridad y el desarrollo, los Estados Miembros deberían convenir en reemplazar la Comisión de Derechos Humanos por un Consejo de Derechos Humanos de carácter permanente y composición más reducida. Los Estados Miembros deberían decidir si desean que el Consejo de Derechos Humanos sea un órgano principal de las Naciones Unidas o un órgano subsidiario de la Asamblea General, 
pero en ambos casos sus miembros serían elegidos directamente por la Asamblea General por el voto de una mayoría de dos tercios de los miembros presentes y votantes. La creación del Consejo situaría a los derechos humanos en una posición de mayor autoridad, acorde con la primacía que se atribuye a los derechos humanos en la Carta de las Naciones Unidas. Los Estados Miembros deberán determinar la composición del Consejo y la duración del mandato de sus miembros. Los miembros del Consejo deberían comprometerse a acatar las normas de derechos humanos más rigurosas.

\section{La Secretaría}

184. Una Secretaría competente y eficaz es indispensable para la labor de las Naciones Unidas. Si las necesidades de la Organización han cambiado, también debe cambiar la Secretaría. Es por ese motivo que en 1997 puse en marcha un conjunto de reformas estructurales para la Secretaría, seguidas en 2002 por una serie de mejoras administrativas y técnicas, encaminadas a dotar a la Organización de un programa de trabajo más preciso y un sistema más sencillo de planificación y presupuestación y permitir que la Secretaría proporcionara mejores servicios.

185. Celebro que la Asamblea General haya prestado un amplio apoyo a esos cambios, que considero que han mejorado nuestra capacidad de realizar la labor que el mundo espera de nosotros. Gracias a las modificaciones en materia de presupuestación, adquisiciones, gestión de los recursos humanos y apoyo a las misiones de mantenimiento de la paz, ahora trabajamos de una forma nueva y distinta. Sin embargo, esas reformas no son suficientes. Para que las Naciones Unidas sean verdaderamente eficaces, la Secretaría debe ser objeto de una completa transformación.

186. Quienes tienen la facultad de adoptar decisiones -fundamentalmente la Asamblea General y el Consejo de Seguridad- deben asegurarse de que, cuando asignen mandatos a la Secretaría, también proporcionen los recursos necesarios para las tareas encomendadas. A cambio, la administración debe ser más responsable de sus actos y debe reforzarse la capacidad de supervisión de los órganos intergubernamentales. Deben conferirse al Secretario General y a su personal directivo las facultades, los medios, la autoridad y la asistencia de expertos que necesiten para administrar una organización que se espera que atienda necesidades operacionales rápidamente cambiantes en muchas partes del mundo. Paralelamente, los Estados Miembros deben disponer de los instrumentos de supervisión que necesiten para garantizar que el Secretario General rinda cuentas verdaderamente de su estrategia y su labor directiva.

187. Los Estados Miembros también tienen que una función esencial que desempeñar para asegurar que los mandatos de la Organización se mantengan actualizados. Por consiguiente, pido a la Asamblea General que examine todos los mandatos de más de cinco años de antigüedad para comprobar si las actividades previstas siguen siendo verdaderamente 
necesarias y si los recursos asignados para su realización pueden redistribuirse para responder a nuevos desafíos.

188. Hoy día, el personal de las Naciones Unidas debe: a) estar en condiciones de hacer frente a los nuevos desafíos sustantivos del siglo $\mathrm{XXI}$; b) contar con los medios necesarios para dirigir operaciones complejas en el plano mundial, y c) estar obligado a rendir cuentas.

189. En primer lugar, me propongo adoptar medidas para adecuar la estructura de la Secretaría a las prioridades expuestas en el presente informe. Ello supondrá crear una oficina de apoyo a la consolidación de la paz y fortalecer el apoyo prestado tanto en favor de la mediación (mi función de "buenos oficios") como en favor de la democracia y el imperio de la ley. Además, tengo la intención de designar a un Asesor Científico del Secretario General, que prestará asesoramiento científico de carácter estratégico y orientado al futuro sobre asuntos normativos movilizando los conocimientos científicos y tecnológicos existentes en el sistema de las Naciones Unidas y en la comunidad científica y académica en general.

190. Para hacer verdaderos progresos en nuevos ámbitos debemos contar con un personal que posea los conocimientos y la experiencia necesarios para afrontar nuevos desafíos. También es necesario redoblar los esfuerzos por alcanzar "el más alto grado de eficiencia, competencia e integridad", como exige el Artículo 101.3 de la Carta de las Naciones Unidas, y al mismo tiempo "contratar el personal en forma de que haya la más amplia representación geográfica posible", a lo que hoy debemos agregar la exigencia de lograr un justo equilibrio entre hombres y mujeres. Aunque el personal existente debe disponer de oportunidades razonables de avanzar en su carrera dentro de la Organización, no podemos seguir recurriendo al mismo grupo de personas para atender todas nuestras nuevas necesidades. Por consiguiente, pido a la Asamblea General que me confiera la autoridad y los recursos necesarios para ofrecer a los funcionarios, a título excepcional, una gratificación por retiro voluntario a fin de renovar y reorientar al personal para atender las necesidades actuales.

191. En segundo lugar, deben proporcionarse a la Secretaría los medios necesarios para realizar su labor. El Grupo de alto nivel propuso que yo nombrara un segundo Vicesecretario General para mejorar el proceso para tomar decisiones en materia de paz y seguridad. En lugar de ese cargo, he decidido crear un mecanismo de adopción de decisiones en forma de gabinete (con mayor poder ejecutivo que el actual Grupo Superior de Gestión) para mejorar la formulación de políticas y la gestión. Contará con el apoyo de una pequeña secretaría que se ocupará de la preparación y el seguimiento de las decisiones. Considero que de ese modo el proceso de adopción de decisiones tendrá objetivos mejor definidos y será más metódico, y también será más clara la asignación de la responsabilidad. Esas medidas serán de utilidad, pero no bastarán por sí solas para lograr una gestión eficaz de las operaciones mundiales de una Organización tan compleja como ésta. El Secretario General, el funcionario administrativo más alto de la Organización, debe tener más 
autoridad y flexibilidad en materia de gestión. Debe tener la capacidad de modificar la plantilla cuando sea necesario sin verse sometido a restricciones excesivas. Además, nuestro sistema administrativo debe ser modernizado totalmente. Por consiguiente, pido a los Estados Miembros que colaboren conmigo para realizar un examen a fondo de las normas relativas al presupuesto y los recursos humanos que rigen nuestras actividades.

192. En tercer lugar, debemos seguir aumentando la transparencia y mejorando la rendición de cuentas en la Secretaría. La Asamblea General ha dado un importante paso para lograr una mayor transparencia al poner las auditorías internas a disposición de los Estados Miembros que lo soliciten. Estoy en vías de determinar qué otras categorías de información podrían ponerse regularmente a disposición de los interesados. Me propongo establecer una Junta de Evaluaciones del Desempeño de las Funciones Directivas para asegurar que los funcionarios superiores rindan cuentas de sus actos y de los resultados de la labor de las dependencias a su cargo. Hay varias mejoras internas en marcha para adoptar en nuestros sistemas de gestión y nuestras políticas de recursos humanos las mejores prácticas de otras organizaciones públicas y comerciales mundiales. A fin de reforzar la rendición de cuentas y la labor de supervisión, he propuesto que la Asamblea General disponga la realización de un examen a fondo de la Oficina de Servicios de Supervisión Interna, con miras a fortalecer su independencia y su autoridad, así como sus conocimientos y su capacidad. Espero que la Asamblea adopte con prontitud una decisión sobre esta propuesta.

\section{Coherencia del sistema}

193. Aparte de la Secretaría, el sistema de fondos, programas y organismos especializados de las Naciones Unidas posee un extraordinario acervo de conocimientos y recursos que abarcan toda la gama de cuestiones de interés mundial. Y lo que es cierto para las Naciones Unidas propiamente dichas también lo es para las demás entidades del sistema. Todas ellas deben tener la clara obligación de rendir cuentas tanto a sus órganos rectores como a las personas a las que prestan servicio.

194. Como hecho positivo, cabe señalar que en los últimos decenios, en respuesta a una demanda en constante aumento, ha aumentado el número de miembros del sistema y la escala y el alcance de sus actividades. Un efecto secundario negativo de esa expansión ha sido la considerable duplicación de mandatos y actividades de los diferentes órganos del sistema, que ocurre con frecuencia en la actualidad. Otro efecto han sido las graves dificultades para obtener la financiación necesaria.

195. Para tratar de resolver algunos de esos problemas, he puesto en marcha dos series de importantes reformas durante mi mandato como Secretario General. En primer lugar, en mi informe de 1997 titulado "Renovación de las Naciones Unidas: un programa de reforma" (A/51/ 950), describí varias medidas que había adoptado, entre las que cabe mencionar la creación de comités ejecutivos destinados a fortalecer la 
capacidad directiva de la Secretaría y facilitar la coordinación de las actividades humanitarias y de desarrollo. Posteriormente, en 2002, en un segundo informe titulado "Fortalecimiento de las Naciones Unidas: un programa para profundizar el cambio" (A/57/387 y Corr. 1 ), expuse nuevas medidas, encaminadas más directamente a mejorar nuestra labor a nivel de los países, especialmente fortaleciendo el sistema de coordinadores residentes. También he conferido más atribuciones a mis representantes especiales y he instituido un sistema de operaciones de paz integradas.

196. Esas iniciativas han producido importantes beneficios, ya que han permitido a los distintos organismos colaborar más estrechamente entre sí y con otros asociados, como el Banco Mundial, en el plano nacional. No obstante, el sistema de las Naciones Unidas en su conjunto todavía no presta servicios de la forma coherente y eficaz que necesita y merece la ciudadanía mundial.

197. Parte del problema tiene que ver claramente con las limitaciones estructurales con que tropezamos. A mediano y largo plazo debemos considerar la posibilidad de reformas mucho más radicales para superarlas. Las reformas podrían entrañar el agrupamiento de los distintos organismos, fondos y programas en entidades rigurosamente administradas, que se ocuparían respectivamente del desarrollo, el medio ambiente y las actividades humanitarias. Esa reagrupación podría suponer la eliminación o la fusión de los fondos, programas u organismos que tuvieran mandatos y competencias complementarias o parcialmente coincidentes.

198. Entretanto, hay medidas más inmediatas que podemos y debemos adoptar desde ahora. En particular, me propongo introducir nuevas mejoras para coordinar la presencia y el desempeño del sistema de las Naciones Unidas en el plano nacional, sobre la base de un sencillo principio: en cada etapa de las actividades de las Naciones Unidas, el alto funcionario de las Naciones Unidas presente en el país de que se trate -representante especial, coordinador residente o coordinador de asuntos humanitarios- debe tener la autoridad y los recursos necesarios para administrar una misión integrada o una "presencia" integrada de las Naciones Unidas en el país, de modo que las Naciones Unidas puedan funcionar verdaderamente como una única entidad integrada.

\section{Las Naciones Unidas en el plano nacional}

199. En todos los países donde las Naciones Unidas realizan actividades de desarrollo, sus organismos, fondos y programas deberían organizar su labor técnica para ayudar al país a formular y aplicar las estrategias nacionales de reducción de la pobreza basadas en los objetivos de desarrollo del Milenio que se detallan en la sección II. Aunque la administración del sistema de coordinadores residentes debe seguir siendo responsabilidad del Programa de las Naciones Unidas para el Desarrollo (PNUD), que es nuestra principal institución en este ámbito, el Grupo de las Naciones Unidas para el Desarrollo (GNUD) debería ocuparse de la 
orientación general de los equipos de las Naciones Unidas en los países, dirigidos por coordinadores residentes que contaran con los recursos y medios apropiados. El Marco de Asistencia de las Naciones Unidas para el Desarrollo debería establecer claros objetivos estratégicos y definir la asistencia específica que cada entidad de las Naciones Unidas ha de prestar para ayudar a nuestros asociados nacionales a alcanzar los objetivos del Milenio y satisfacer sus necesidades más generales en materia de desarrollo. Tanto los gobiernos como la propia Organización pueden utilizar posteriormente esta "matriz de resultados" para supervisar y evaluar la actuación del sistema de las Naciones Unidas a nivel nacional y pedir cuentas a sus representantes.

\section{Fortalecimiento del sistema de coordinadores residentes}

200. Para impulsar este proceso, seguiré fortaleciendo la función de los coordinadores residentes, otorgándoles más autoridad para que puedan mejorar su labor de coordinación. Pero los órganos rectores de los distintos organismos también tienen que impartir orientación para apoyar este proceso. Pido a los Estados Miembros que coordinen la labor de sus representantes en esos órganos rectores, para asegurarse de que sigan una política coherente al asignar mandatos y recursos en todo el sistema. También insto a los Estados Miembros a que aumenten la financiación básica y reduzcan la proporción de fondos destinados a fines específicos para ayudar a mejorar la coherencia del sistema. Como he dicho, espero que, tras su revitalización, el Consejo Económico y Social pueda dar orientación general a esta nueva coherencia.

201. En los últimos años he observado con satisfacción los beneficios que el sistema de las Naciones Unidas ha obtenido al colaborar estrechamente con científicos independientes, responsables de la formulación de políticas y dirigentes políticos de todo el mundo. Así ocurre sobre todo en el ámbito del desarrollo, donde constantemente es necesario integrar los últimos avances de la ciencia y la tecnología en la práctica de nuestros programas y organizaciones. En 2005 me propongo crear un consejo de asesores en cuestiones de desarrollo a fin de consolidar los vínculos entre la labor de desarrollo de las Naciones Unidas y los principales expertos mundiales en las esferas pertinentes. Este consejo, que trabajará en estrecha cooperación con el Asesor Científico del Secretario General ya mencionado, estará integrado por una veintena de personas: destacados científicos, altos funcionarios y líderes políticos del mundo. El Consejo nos asesorará tanto a mí como al GNUD sobre la mejor manera de respaldar la consecución de los objetivos de desarrollo del Milenio, publicará periódicamente informes y comentarios y servirá de enlace con la comunidad científica, la sociedad civil y otros órganos que cuenten con los conocimientos especializados pertinentes. El Consejo Económico y Social también podrá pedirle asesoramiento.

\section{Sistema de respuesta humanitaria}

202. Durante los últimos meses se ha puesto claramente de manifiesto la creciente magnitud y variedad de las exigencias que el mundo plantea al 
sistema internacional de respuesta humanitaria, en casos como la tragedia del tsunami del Océano Índico o las crisis en Darfur y la parte oriental de la República Democrática del Congo. Gracias al liderazgo y a la coordinación de las Naciones Unidas, el sistema integrado por la comunidad humanitaria de organismos y organizaciones no gubernamentales está desempeñando bastante bien su labor, dadas las circunstancias. En cuestión de días se despliegan expertos trabajadores humanitarios en cualquier sitio del mundo y se distribuyen entre las víctimas de la guerra y los desastres naturales grandes cantidades de alimentos y otros suministros de socorro. Se ha reducido la superposición de la labor de los distintos organismos y ha aumentado la eficacia de la coordinación entre las entidades no gubernamentales e intergubernamentales sobre el terreno.

203. El sistema logró ofrecer asistencia de socorro en masa a todas las comunidades afectadas por el tsunami en el Océano Índico, pese a las circunstancias adversas, en el transcurso de unas semanas. Pero, al mismo tiempo, la asistencia prestada a los desplazados de Darfur dista mucho de haber alcanzado el nivel prometido y, lamentablemente, sigue sin haber fondos suficientes para responder a graves crisis como la de la República Democrática del Congo, donde más de 3,8 millones de personas han resultado muertas y 2,3 millones desplazadas desde 1997. La respuesta humanitaria tiene que ser más previsible en todas las situaciones de emergencia y para lograrlo es preciso avanzar rápidamente en tres frentes.

204. En primer lugar, el sistema humanitario debe contar con una capacidad de respuesta más previsible en ciertas esferas, desde el suministro de agua y el saneamiento hasta el alojamiento y la administración de los campamentos, en las que actualmente hay demasiadas deficiencias. Cuando ya se ha iniciado una crisis, hay que actuar con prontitud y flexibilidad. Esto es muy evidente en el caso de las situaciones de emergencia complejas, donde las necesidades humanitarias guardan relación con la dinámica del conflicto y las circunstancias pueden cambiar rápidamente. En general, los equipos de las Naciones Unidas en los países, bajo la dirección del coordinador de asuntos humanitarios, son los que están en mejores condiciones de detectar las oportunidades y dificultades existentes. Sin embargo, es claramente necesario reforzar las estructuras de coordinación sobre el terreno, en especial preparando y dotando mejor a los equipos de las Naciones Unidas en los países, fortaleciendo el liderazgo del coordinador de asuntos humanitarios y asegurándose de que existan recursos suficientes y flexibles para apoyar de inmediato a estas estructuras sobre el terreno.

205. En segundo lugar, hay que contar con financiación previsible para satisfacer las necesidades de las comunidades vulnerables. Es preciso que el generoso apoyo mundial prestado durante la crisis del tsunami sea la regla y no la excepción. Para ello hay que aprovechar la colaboración de la comunidad humanitaria con los donantes y lograr 
una participación más sistemática de nuevos gobiernos donantes y del sector privado. Para asegurar una respuesta coherente y oportuna a las crisis es necesario que se proporcionen rápidamente los recursos prometidos y también que se destinen fondos en forma más previsible y flexible a las operaciones humanitarias, particularmente en las etapas iniciales de emergencia.

206. En tercer lugar, es necesario que el derecho de acceso de nuestros trabajadores humanitarios y operaciones sobre el terreno sea previsible y que su seguridad esté garantizada. Con demasiada frecuencia se impide al personal humanitario prestar asistencia debido a que las fuerzas del gobierno o los grupos armados obstaculizan su labor. En otros casos, los terroristas atacan a nuestros trabajadores inermes y paralizan sus operaciones de ayuda, infringiendo los principios básicos del derecho internacional.

207. Estoy trabajando con el Coordinador del Socorro de Emergencia a fin de resolver estos problemas y formular recomendaciones concretas para adoptar medidas más enérgicas. En estos momentos se está realizando un examen a fondo de la respuesta humanitaria, cuyas conclusiones se darán a conocer en junio de 2005. Espero que dichas conclusiones incluyan una serie de propuestas para establecer nuevos acuerdos de personal y equipo de reserva a fin de garantizar la capacidad de responder inmediatamente a grandes desastres y otras situaciones de emergencia, si es preciso en varias zonas a la vez. Estoy decidido a colaborar con los Estados Miembros y los organismos pertinentes para que estas propuestas, una vez finalizadas, se apliquen sin dilación.

208. Para poder responder de inmediato a los desastres repentinos o a las acuciantes necesidades que quedan insatisfechas cuando no se presta suficiente atención a una situación de emergencia, tenemos que considerar si los instrumentos financieros de que disponemos son adecuados. Habría que determinar si se debe actualizar el Fondo Renovable Central para Emergencias o si es necesario establecer un nuevo mecanismo de financiación. En este último caso, convendría estudiar seriamente la propuesta hecha por los donantes de crear un fondo de contribuciones voluntarias por valor de 1.000 millones de dólares.

209. Hay que prestar especial atención al creciente problema de los desplazados. Al contrario de lo que ocurre con los refugiados, que han atravesado una frontera internacional, las personas desplazadas dentro de su propio país por la violencia y la guerra no cuentan con la protección de las normas mínimas establecidas.

210. Sin embargo, este grupo sumamente vulnerable está integrado en la actualidad por uno 25 millones de personas, más del doble que el número estimado de refugiados. Insto a los Estados Miembros a que aprueben los Principios rectores aplicables a los desplazamientos dentro del país (E/CN.4/1998/53/Add.2), preparados por mi Representante Especial, como norma básica internacional para proteger a esas personas, y a 
que se comprometan a promover la aplicación de estos principios mediante la legislación nacional. A diferencia de los refugiados, de quienes se ocupa la Oficina del Alto Comisionado de las Naciones Unidas para los Refugiados, las personas desplazadas ven con frecuencia cómo sus necesidades quedan insatisfechas por no estar comprendidas exactamente en el mandato de ningún organismo humanitario. Recientemente se han tomado medidas para asegurar que los organismos colaboren para prestar asistencia a estos grupos dentro de sus respectivos ámbitos de competencia. Pero, como hemos comprobado no hace mucho en Darfur, es necesario redoblar los esfuerzos. Tengo intención de seguir fortaleciendo la respuesta interinstitucional a fin de atender a las necesidades de los desplazados internos, bajo la dirección del Coordinador del Socorro de Emergencia a nivel mundial y mediante el sistema de coordinadores de asuntos humanitarios a nivel nacional. Espero contar con el apoyo de los Estados Miembros en esta iniciativa.

211. Por último, tengo la intención de pedir de forma más sistemática a los Estados Miembros en general y al Consejo de Seguridad en particular que se ocupen del problema que plantean los inaceptables bloqueos del acceso humanitario con que nos encontramos demasiado a menudo. Para evitar sufrimientos innecesarios, es esencial proteger el espacio humanitario y asegurar que las instituciones humanitarias tengan acceso a las poblaciones vulnerables, sin trabas y en condiciones de seguridad. También tomaré medidas, mediante el Departamento de Seguridad y Vigilancia que acaba de crearse en la Secretaría, para reforzar nuestro sistema de gestión de riesgos, a fin de que los trabajadores humanitarios puedan llevar a cabo sus operaciones vitales en zonas muy peligrosas sin arriesgar innecesariamente su vida.

\section{Gobernanza del medio ambiente mundial}

212. El sector del medio ambiente plantea problemas especiales de coherencia, debido al número y la complejidad de los acuerdos y organismos internacionales que se ocupan de él. Actualmente están en vigor más de 400 tratados multilaterales sobre el medio ambiente de alcance regional y universal, que abarcan una amplia gama de cuestiones, como la biodiversidad, el cambio climático y la desertificación. El carácter sectorial de estos instrumentos jurídicos, así como la fragmentación de los mecanismos utilizados para supervisar su aplicación, dificultan aún más la organización de respuestas eficaces en todos los casos. Es claramente necesario racionalizar y unificar nuestros esfuerzos por aplicar estos tratados. Ya en 2002, durante la Cumbre Mundial sobre el Desarrollo Sostenible celebrada en Johannesburgo, se subrayó la necesidad de contar con un marco institucional más coherente para la gobernanza internacional del medio ambiente, con una mejor coordinación y supervisión. Ha llegado el momento de considerar la posibilidad de crear una estructura más integrada para establecer normas ambientales, debatir cuestiones científicas y supervisar el cumplimiento de los tratados. Para ello deberían utilizarse las instituciones existentes, como el Programa de las Naciones Unidas para el Medio Ambiente, así como 
los órganos creados en virtud de tratados y los organismos especializados. Entretanto, las actividades ambientales realizadas a nivel nacional saldrían beneficiadas si los organismos de las Naciones Unidas intensificaran sus relaciones sinérgicas, tanto en los aspectos normativos como operacionales, aprovechando al máximo sus ventajas comparativas a fin de lograr un enfoque integrado del desarrollo sostenible que conceda la debida importancia a los dos elementos de este concepto.

\section{E. Organizaciones regionales}

213. Existe en la actualidad un número considerable de organizaciones regionales y subregionales en todo el mundo que hacen importantes contribuciones a la estabilidad y prosperidad de sus miembros y del sistema internacional en su conjunto. Las Naciones Unidas y las organizaciones regionales deberían complementarse para hacer frente a los problemas que ponen en peligro la paz y la seguridad internacionales. A este respecto, los países donantes deberían prestar especial atención a la necesidad de establecer un plan decenal para el fomento de la capacidad conjuntamente con la Unión Africana. Para mejorar la coordinación entre las Naciones Unidas y las organizaciones regionales, dentro del marco de la Carta, tengo intención de establecer memorandos de entendimiento entre las Naciones Unidas y cada una de esas organizaciones a fin de regular el intercambio de información, conocimientos especializados y recursos, según los casos. En cuanto a las organizaciones regionales que están en condiciones de realizar actividades de prevención de conflictos o mantenimiento de la paz, estos memorandos de entendimiento podrían servir para integrar dichas actividades en el marco del Sistema de Acuerdos de Fuerzas de Reserva de las Naciones Unidas.

214. También tengo intención de invitar a las organizaciones regionales a participar en las reuniones de los órganos de coordinación del sistema de las Naciones Unidas cuando se debatan cuestiones en las que estén particularmente interesadas.

215. Deberían enmendarse las normas relativas a los presupuestos de las operaciones de mantenimiento de la paz de las Naciones Unidas para que la Organización pueda optar, en circunstancias muy excepcionales, por utilizar las cuotas para financiar las operaciones regionales autorizadas por el Consejo de Seguridad o la participación de las organizaciones regionales en operaciones de paz multilaterales llevadas a cabo bajo la dirección general de las Naciones Unidas.

\section{F. Actualización de la Carta de las Naciones Unidas}

216. Como señalé al comienzo de la sección $V$, los principios de la Carta de las Naciones Unidas siguen teniendo plena validez y la propia Carta continúa, en esencia, ofreciendo un sólido fundamento para toda nuestra labor. Básicamente se trata del mismo documento que se redactó en la Conferencia de San Francisco hace 60 años. Se ha avanzado mucho efectuando modificaciones en la práctica sin necesidad de enmendar el 
documento. De hecho, la Carta sólo ha sido enmendada dos veces durante la historia de la Organización, cuando se decidió ampliar el número de miembros del Consejo de Seguridad y del Consejo Económico y Social.

217. Sin embargo, las Naciones Unidas operan hoy en un mundo que es completamente distinto del que existía en 1945, y la Carta debería reflejar las realidades de nuestros días. En particular, ha llegado el momento de suprimir las referencias anacrónicas a los Estados "enemigos" contenidas en los Artículos 53 y 107.

218. El Consejo de Administración Fiduciaria desempeñó una función vital para mejorar la administración de los territorios en fideicomiso y promover el proceso de descolonización en su conjunto. No obstante, hace mucho tiempo que concluyó su labor. Por lo tanto, debería suprimirse el Capítulo XIII de la Carta, titulado "Consejo de Administración Fiduciaria".

219. Por razones análogas, debería eliminarse el Artículo 47, relativo al Comité de Estado Mayor, así como todas las referencias a este Comité contenidas en los Artículos 26, 45 y 46. 